Iully Crislaine Cunha Andrade ${ }^{1}$

Carla Kalline Alves Cartaxo Freitas ${ }^{1}$

Maria do Socorro Claudino Barreiro ${ }^{1}$

Andreia Freire de Menezes ${ }^{1}$

\section{Demographic and clinical profile of workers assisted at a Reference Occupational Health Center}

\title{
Perfil demográfico e clínico de trabalhadores atendidos em um Centro de Referência em Saúde do Trabalhador
}

ABSTRACT | Introduction: The bealth of the worker encompasses the field of knowledge that seeks to understand the relationships between work and the health/disease process. Objective: To describe the demographic and clinical profile of workers assisted at Reference Occupational Health Center (CEREST) in Lagarto County - Sergipe State. Methods: Cross-sectional descriptive study based on a quantitative approach; it was developed based on secondary data analysis; data were collected in duly completed records of patients assisted at CEREST between

January 2013 and September 2016.

Results: $63 \%$ of the assessed workers were male, $54 \%$ were in the age group $30-49$ years, $67 \%$ were born in Lagarto County, $60 \%$ were farmers. In total, $42 \%$

of workers' complaints regarded bone issues,

beadache, nausea and vomiting; $16 \%$ had syncope and $11 \%$ reported neuromuscular issues. Most medical conducts referred patients to specialties; orthopedic and dermatological issues stood out among them: $53 \%$ and $11 \%$, respectively. Conclusion:

Demographic and clinical characteristics of the working class assisted at CEREST were identified; however, records showed normalization deficiency, which impaired the observation of all mainly variables to be investigated. Research studies often aim at identifying workers needs and at developing interventions to improve the context experienced by local populations.

Keywords | Health of workers; Health surveillance; Health profile.
RESUMO | Introdução: A saúde do trabalhador abrange o campo do saber que busca a compreensão das relações existentes entre o trabalho e o processo saúde/ doença. Objetivo: Descrever o perfil demográfico e clínico de trabalhadores atendidos em um Centro de Referência em Saúde do Trabalhador (CEREST). Métodos: Trata-se de um estudo transversal, descritivo de abordagem quantitativa, desenvolvido mediante análise de dados secundários, obtidos a partir de prontuários devidamente preenchidos da população atendida no CEREST entre janeiro de 2013 e setembro de 2016. Resultados: Observou-se que 63\% dos trabalhadores eram do sexo masculino, $54 \%$ se encontravam na faixa etária entre 30-49 anos, $67 \%$ nasceram no município de Lagarto, $60 \%$ possuíam a ocupação de lavrador, $42 \%$ das queixas dos trabalhadores foram ósseas, seguidas de cefaleia, náuseas, vômitos, síncope $16 \%$ e neuromusculares $11 \%$. A conduta médica mais frequente foi o encaminhamento a especialidades com $53 \%$, e dentre elas destacam-se, a ortopedia (54\%) e dermatologia (11\%). Conclusão: Foram identificadas características demográficas e clínicas da classe trabalhadora atendida no CEREST Lagarto, no entanto observou-se uma deficiência na normatização dos registros, a qual dificultou a contemplação de todas as variáveis que o estudo visava investigar. Estudos de investigação servem para identificar necessidades e desenvolver intervenções com o intuito de melhorar o contexto no qual a população local está inserida.

Palavras-chave| Saúde do trabalhador; Vigilância em saúde; Perfil de saúde.

\footnotetext{
${ }^{1}$ Universidade Federal de Sergipe. Aracaju/SE, Brasil.
} 


\section{INTRODUÇÃO|}

A saúde do trabalhador abrange o campo do saber que busca a compreensão das relações existentes entre o trabalho e o processo saúde/doença ${ }^{1}$. E levando-se em conta as condições em que ele é exercido, o trabalho pode atuar tanto para melhorias nas habilidades humanas quanto para o adoecimento, agravamento do adoecer e até mesmo para a redução dos anos de vida dos trabalhadores². No Brasil, a saúde do trabalhador é considerada uma competência do Sistema Único de Saúde (SUS), que passou a ser garantida a partir de 1988 com a Constituição Federal e foi então consolidada pela Lei no $8.080 / 90^{3}$.

É importante conhecer, identificar e controlar os riscos ocupacionais aos quais os trabalhadores tradicionalmente estão expostos: riscos físicos (ruído, frio e calor, vibração etc.), químicos (agentes e substâncias químicas na forma líquida, gasosa, ou de partículas e de poeiras minerais ou vegetais), biológicos (vírus, bactérias, parasitas). Além desses, os riscos ergonômicos e psicossociais (uso de máquinas e mobiliário inadequados, más condições de iluminação, ventilação e de conforto, monotonia, relações de trabalho autoritárias etc.), e os riscos mecânicos e de acidentes, relacionados ao arranjo físico, protetores em máquinas, ordem e limpeza no ambiente, sinalização, entre outros que podem levar a acidentes de trabalho ${ }^{2}$.

No entanto, algo que muitas vezes incentiva o descumprimento de disposições elementares para prevenção dos riscos profissionais, acidentes de trabalho e doenças ocupacionais, é a determinação pelo mercado trabalhista de relações desproporcionais entre a procura e a oferta de trabalho. O fato é que nos dias de hoje, a circulação de bens e pessoas encontra-se muito facilitada, e isso abre portas para uma concorrência desenfreada que constitui um sério obstáculo à saúde e à segurança do trabalho ${ }^{4}$.

Essa perspectiva de defesa da saúde dos trabalhadores deve atuar em consonância com as políticas públicas voltadas à saúde ocupacional no Brasil. Em 2002 foi instituída pela Portaria no 1.679 a Rede Nacional de Atenção Integral à Saúde do Trabalhador (RENAST), considerando que ações de prevenção, promoção e recuperação da saúde dos trabalhadores urbanos e rurais fossem desenvolvidas no âmbito do SUS ${ }^{5}$. Posteriormente, a RENAST foi revista pela Portaria no 2.728 de 11 de novembro de 2009, a qual determina que as ações em saúde do trabalhador devem ser desenvolvidas de forma descentralizada e hierarquizada ${ }^{6}$.
Com efeito, a implementação da RENAST se dá, dentre outras formas, por meio da estruturação da rede de Centros de Referência em Saúde do Trabalhador (CEREST), os quais têm a função de dar subsídio técnico ao SUS nas ações de promoção, prevenção, vigilância, diagnóstico, tratamento e reabilitação da saúde dos trabalhadores urbanos e rurais ${ }^{6}$. Ao CEREST cabe a atribuição de funcionamento como polos irradiadores da cultura da produção social das doenças, e do trabalho como centro desse processo. É de sua competência realizar o provimento de suporte técnico e informações, viabilizando as ações de vigilância, além de executar, organizar e estruturar a assistência de média e alta complexidade relacionada com problemas e agravos à saúde associados ao trabalho ${ }^{7}$.

O conhecimento de características da classe trabalhadora faz-se importante, pois a principal referência para se entender as condições de vida, o perfil de adoecimento e morte dos indivíduos, corresponde à inter-relação entre trabalho, ambiente e saúde, determinada pelo modo de produção e consumo de uma sociedade. Do mesmo modo, essa inter-relação é referência para que sejam geradas alternativas de mudança capazes de garantir vida e saúde para a população trabalhadora ${ }^{8}$. E é no contexto das atribuições do CEREST que o presente estudo teve como objetivo descrever o perfil demográfico e clínico dos trabalhadores atendidos no Centro de Referência em Saúde do Trabalhador Regional do município de Lagarto - SE.

\section{MÉTODOS|}

Trata-se de um estudo transversal, descritivo de abordagem quantitativa, desenvolvido mediante análise de dados secundários. A pesquisa foi realizada no CEREST regional de Lagarto, localizado no interior do estado de Sergipe, que abrange além desse município, os municípios de Riachão do Dantas, Poço Verde, Simão Dias, Salgado e Tobias Barreto. O referido CEREST desempenha em sua rotina, atividades voltadas à vigilância, com a realização de visitas técnicas a empresas e locais de trabalho, bem como realiza atividades educativas que têm como princípio a defesa da saúde dos trabalhadores. Além disso, recebe os trabalhadores em consultas junto à equipe que o constitui: médico, enfermeiro, fonoaudiólogo, fisioterapeuta e psicólogo.

A amostra do estudo foi obtida a partir dos prontuários da população atendida no CEREST. Foram incluídos na 
pesquisa os prontuários identificados com data entre janeiro de 2013 e setembro de 2016 e excluídos aqueles que apresentavam irregularidades, como a não identificação das datas ou aqueles que de modo geral não atenderam ao objetivo da pesquisa.

Um total de 115 prontuários foram selecionados para compor a amostra do estudo. As informações foram coletadas com auxílio de instrumento de coleta de dados (Apêndice A), que é dividido entre os aspectos sociodemográficos: sexo, faixa etária, naturalidade, escolaridade, estado civil; ocupacionais: ocupação, de acordo com a Classificação Brasileira de Ocupações - CBO, tempo de serviço, situação trabalhista, uso de Equipamentos de Proteção Individual - EPI's; e de morbidade: queixas dos trabalhadores, doenças ou diagnóstico médico e conduta médica.

As informações obtidas foram analisadas por intermédio da estatística descritiva, utilizando-se o programa Excel, foram criados gráficos e tabela para distribuição dos dados. O sigilo das informações coletadas e a preservação da identidade e dos direitos dos sujeitos foram garantidos; a pesquisa segue os preceitos éticos conforme estabelece a Resolução no 466/12 e foi submetida ao Comitê de Ética e Pesquisa com Seres Humanos, com posterior aprovação. Parecer $\mathrm{n}^{\circ} 1.885 .456$.

\section{RESULTADOS}

Observou-se que $63 \% \quad(n=73)$ da população que frequentou o serviço no período da coleta pertenciam ao sexo masculino e que a faixa etária foi variável, prevalecendo a faixa entre $30-49$ anos, com 54\% ( $n=62$ ). Quanto ao aspecto naturalidade, observou-se que em parte dos prontuários essa variável não foi informada, o que representou $28 \%(n=32)$ desses. No entanto, quando informada, notou-se que $67 \%(\mathrm{n}=77)$ dos trabalhadores eram naturais do município de Lagarto, 3\% $(n=3)$ do município de Simão Dias e 3\% $(n=3)$ do município de Salgado; e as demais cidades que constituem a abrangência do CEREST regional não foram identificadas. As variáveis escolaridade e estado civil não foram encontradas descritas nos prontuários.

Tratando-se da situação ocupacional, conforme mostra a Tabela 1, foi possível verificar que a ocupação de maior destaque foi a de lavrador, que representou $60 \%(n=69)$ do total, seguida por $8 \%(\mathrm{n}=9)$ de agente comunitário de saúde (ACS) e $6 \%(n=7)$ de pedreiros, enquanto as demais variaram bastante e representaram pequenos grupos: $3 \%(n=4)$ exerciam as atividades de auxiliar de serviços gerais, $2 \%(n=2)$ de auxiliar de limpeza, 3\% $(n=3)$ eram domésticas, $3 \%(n=3)$ cozinheiras, $3 \%(n=3)$ eram do ramo da carpintaria, e $13 \%(n=15)$ para outras atividades laborais que se apresentaram isoladamente.

Tabela 1 - Distribuição da ocupação dos trabalhadores atendidos no CEREST entre 2013 e 2016

\begin{tabular}{lcc}
\hline Ocupação & N & \% \\
\hline Lavrador & 69 & 60 \\
ACS & 9 & 8 \\
Pedreiro & 7 & 6 \\
Auxiliar de Serviços Gerais & 4 & 3 \\
Auxiliar de Limpeza & 2 & 2 \\
Doméstica & 3 & 3 \\
Cozinheira & 3 & 3 \\
Carpinteiro & 3 & 3 \\
Outros & 15 & 13 \\
\hline Total & $\mathbf{1 1 5}$ & $\mathbf{1 0 0}$ \\
\hline
\end{tabular}

Quanto à situação trabalhista, $68 \% \quad(\mathrm{n}=79)$ dos trabalhadores eram autônomos, 27\% $(n=31)$ empregados e apenas $4 \%(n=5)$ aposentados. O tempo de serviço, não foi informado em 45\% ( $n=52)$ dos registros, nos demais, o período de trabalho que prevaleceu foi menor que 10 anos correspondendo a 18\% $(\mathrm{n}=21)$ dos trabalhadores. No que se refere à variável uso de EPI, observou-se que $23 \%(n=27)$ dos trabalhadores não fazem uso de EPI e $5 \%(n=6)$ fazem uso dos equipamentos, no entanto, $71 \%$ $(\mathrm{n}=82)$ dessa variável não foi informada.

Já no que se refere aos aspectos de morbidade, as queixas mais frequentemente relatadas nos prontuários foram as ósseas, com 42\% $(n=61))$, seguidas por cefaleia, náuseas, vômitos, síncope, $16 \%(\mathrm{n}=23)$ e neuromusculares, com $11 \%(n=16)$, além das lesões de pele, com $8 \%(n=12)$, e de outras conforme mostra Figura 1. A descrição das doenças/ diagnósticos médicos não constava nos prontuários.

No que se refere às condutas médicas, foi observado que $53 \%(n=84)$ dos casos atendidos eram encaminhados a alguma especialidade, em $28 \%$ foram $(n=45)$ solicitados 
Figura 1 - Distribuição das queixas apresentadas pelos trabalhadores atendidos no CEREST entre 2013 e 2016

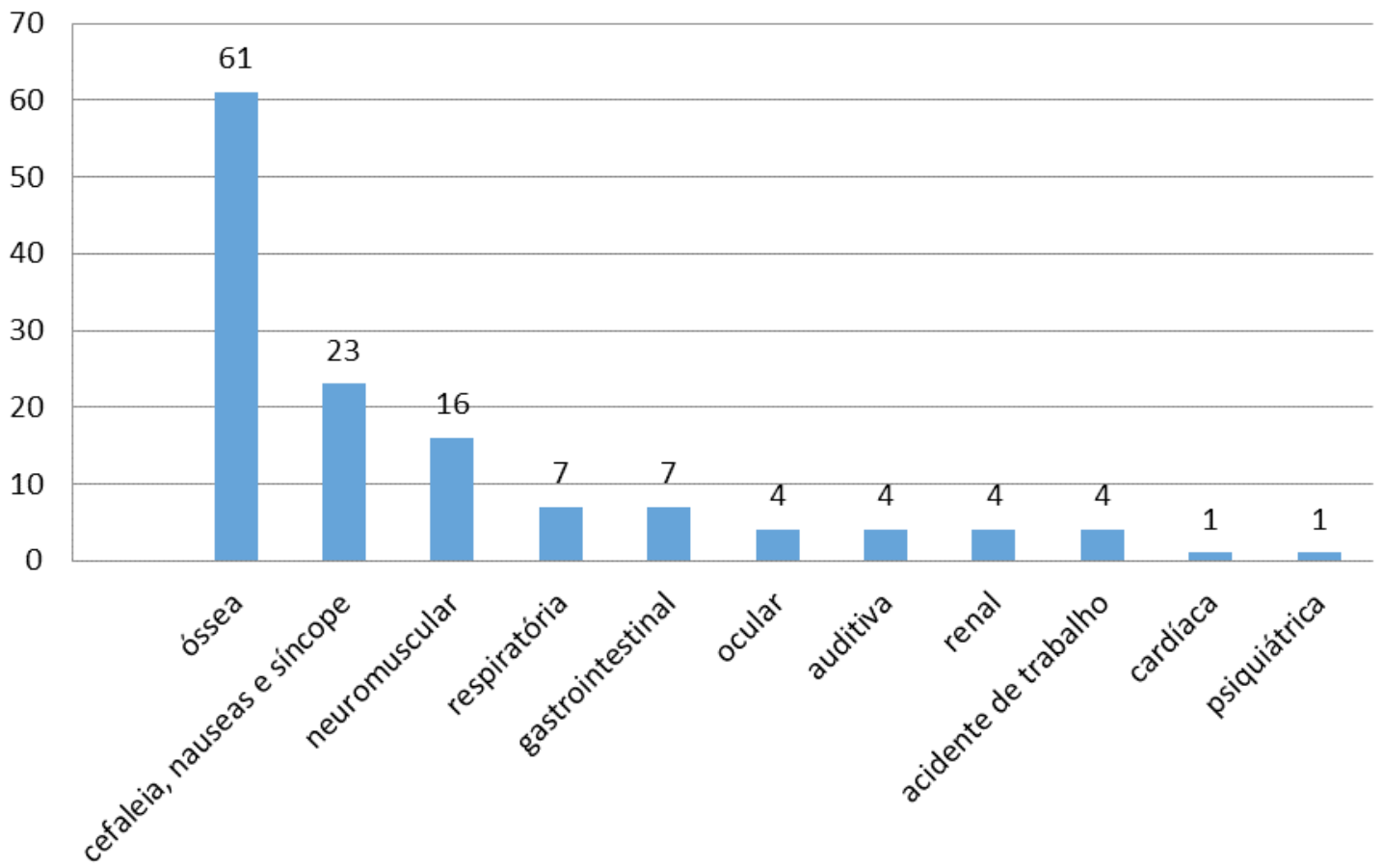

Figura 2 - Distribuição das especialidades às quais foram encaminhados os trabalhadores atendidos no CEREST entre 2013 e 2016

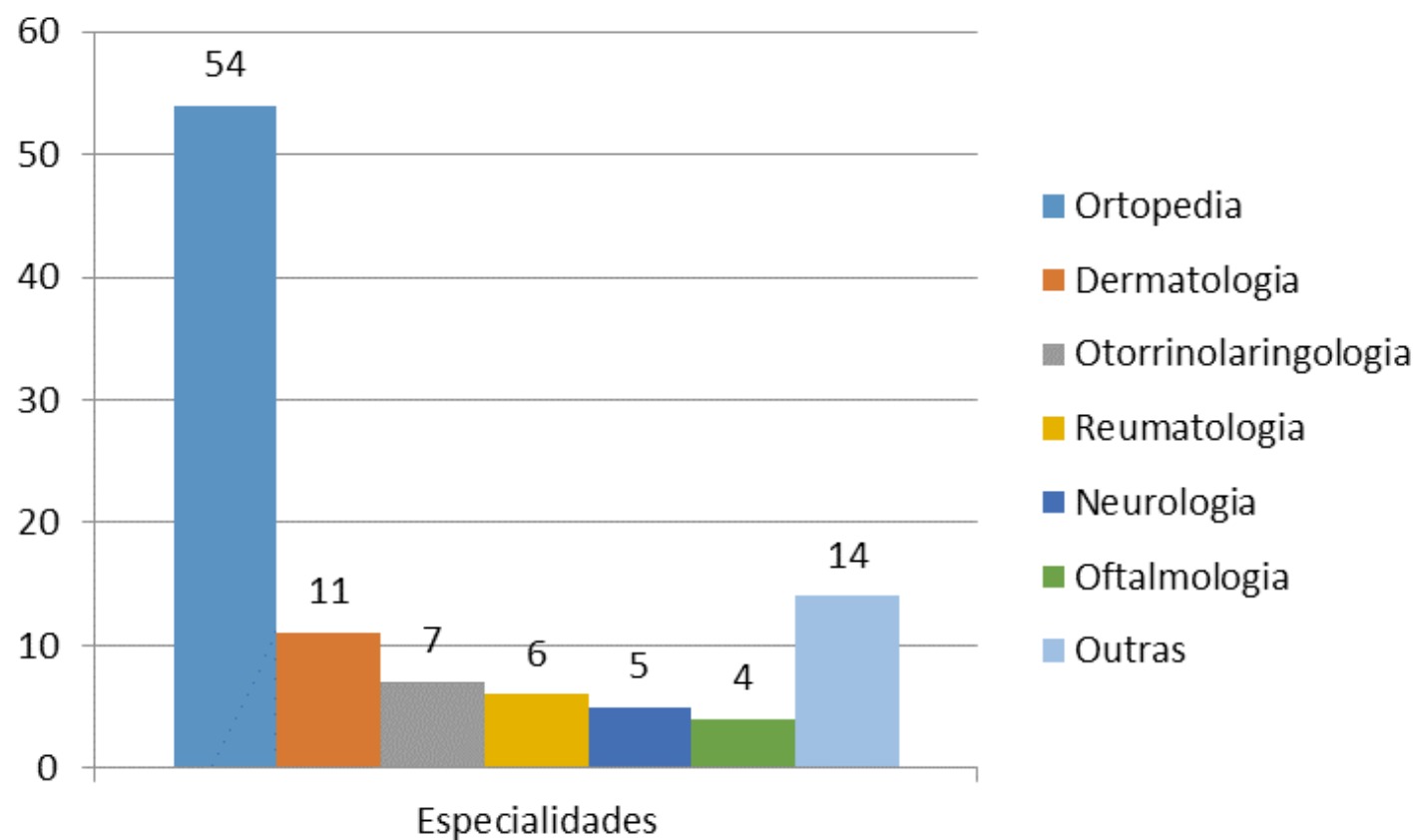

exames laboratoriais e de imagem, e em 11\% $(n=17)$ foi solicitado/emitido relatório ou afastamento do trabalhador. Além disso, algumas outras condutas também foram tomadas, como a prescrição de medicamentos $(4 \%, \mathrm{n}=7)$, orientações $(4 \%, n=6)$ e notificação $(1 \%, n=1)$.
No que se refere às especialidades às quais foram encaminhados os trabalhadores, 54\% $\quad(n=45)$ foram encaminhados à ortopedia, $11 \%(n=9)$ à dermatologia e $7 \%(n=6)$ à otorrinolaringologia. A Figura 2 especifica esses dados. 


\section{DISCUSSÃO|}

Em meio aos resultados, algumas variáveis importantes não estavam descritas nos prontuários, tais como a escolaridade, estado civil e doenças/diagnósticos, o que nos permite inferir uma dificuldade de normatização dos registros. Apesar da falta desses dados, os prontuários foram todos analisados. A falta de informações consiste num fator que pode prejudicar as ações de vigilância em saúde, pois informações que são geradas por prontuários de pacientes, constituem bases de dados que podem subsidiar o planejamento de medidas de prevenção, conforme afirma Carazin?.

Com relação aos aspectos sociodemográficos, observou-se uma prevalência do sexo masculino, em detrimento do sexo feminino, um achado que pode ser cruzado com o tipo de ocupação que ganhou destaque no estudo, a ocupação de lavrador. O mesmo acontece no estudo de Moreira ${ }^{10}$, no qual afirma-se que os trabalhadores com ocupação agrícola são de maioria masculina, quando comparados aos de ocupação não agrícola.

Quanto à naturalidade, evidenciou-se a predominância da população do município de Lagarto. Esse fato pode estar relacionado à localização do CEREST nesse município, embora o serviço seja de abrangência regional e atue também em outras cinco cidades circunvizinhas. Dentro desse contexto, é possível destacar eventuais barreiras de acesso ao serviço do CEREST, às quais a população pode estar submetida, tal como a distância/deslocamento ou até mesmo o não conhecimento a respeito das ações em saúde do trabalhador que o SUS fornece.

No que se refere ao aspecto ocupacional, embora bastante variado, houve um predomínio da ocupação lavrador, seguida por agente comunitário de saúde e pedreiro; a situação trabalhista que predominou foi autônoma, e quanto ao tempo de trabalho, o que prevaleceu foi aquele inferior a 10 anos. Todas essas características refletem um aspecto da região, principalmente no tocante à profissão de lavrador, uma vez que o Nordeste apresenta boa concentração de trabalhadores agrícolas, e a área em que o estudo foi desenvolvido está fortemente ligada ao cultivo, conforme evidenciam alguns estudos ${ }^{10-11}$. A faixa etária entre os 30 e 49 anos ganhou destaque, o que pode estar refletindo a população economicamente ativa, e esse fato constitui um achado que também pode ser visualizado no estudo de Santana ${ }^{12}$, no qual verificou-se uma prevalência da faixa etária de 31 a 50 anos, entre trabalhadores rurais.

Dentre as queixas mais relatadas entre a população estudada, destacaram-se as ósseas, bem como as neuromusculares, fortemente ligadas às condições ergonômicas de trabalho da população que ganhou destaque nesta pesquisa. A ergonomia ainda é pouco aplicada no processo de trabalho rural, devido ao caráter disperso dessa atividade bem como em virtude de equipamentos e máquinas ainda serem quase sempre rudimentares, o que abre portas para a necessidade de aperfeiçoamentos que levem em consideração os fatores ergonômicos e tecnológicos já disponíveis ${ }^{13}$.

Além disso, outro estudo ${ }^{10}$ verificou que ocorre uma prevalência de doenças ósseas, com destaque para afecções da coluna entre a população de trabalhadores agrícola, quando comparados com outras ocupações. Costa et al..$^{13}$ afirmam que atividades em que a enxada é utilizada como instrumento de trabalho, são as que necessitam de intervenção ergonômica, pois o uso incorreto dessa ferramenta submete o trabalhador a uma inclinação anterior do tronco que consequentemente acarreta a deterioração dos discos intervertebrais da região lombar, o que justifica quadros de lombalgia.

Outro destaque foi para as queixas referentes às lesões de pele, as quais são frequentes em meio às ocupações mais prevalentes deste estudo (lavrador, ACS e pedreiro etc.) e que podem estar ligadas a fatores físicos, químicos ou mecânicos. Os ACS, por exemplo, constituem uma classe trabalhadora que exerce sua atividade laboral em meio à exposição solar e, em virtude disso, a face, pescoço, braços, pernas, mãos e olhos são as áreas frequentemente mais expostas e sujeitas a alterações de pele ${ }^{14}$. Embora existam recursos (protetor solar, uso de blusas de manga comprida, bonés, óculos e cuidados com relação ao horário de exposição ao sol), para prevenção de problemas de pele relacionados ao exercício dessa profissão, ainda se sabe pouco a respeito da adesão desses trabalhadores a tais medidas preventivas ${ }^{15}$.

De tal modo ocorre com os lavradores, que por causa do trabalho ao ar livre, esses profissionais expostos à demasiada radiação solar têm alta incidência de câncer de pele, e em média $90 \%$ dessa doença se desenvolve em regiões do corpo expostas ao sol ${ }^{14-16}$. No contexto da prevenção de riscos ocupacionais, verificou-se que, apesar de na grande maioria dos casos esse aspecto não ser informado, existe 
uma taxa muito reduzida de uso de equipamentos de proteção individual por parte dos trabalhadores da região. Um fato que pode estar colocando em risco a saúde dos trabalhadores locais, em sua grande maioria lavradores em contato com agrotóxicos. Esse dado pode ser cruzado com as altas taxas de queixas que evidenciam sinais de intoxicação (cefaleia, náuseas, vômitos, síncope), as quais podem estar ligadas à deficiência do uso de EPIs.

Estudos ${ }^{17-18}$ revelaram que dentre os principais sintomas de intoxicação por agrotóxicos, segundo as queixas de trabalhadores rurais, estavam a cefaleia, irritação na pele e tontura e, que existe entre essa classe trabalhista uma considerável resistência ao uso de EPIs, sob a justificativa de desconforto e alto custo de alguns equipamentos. De maneira geral, os EPIs fazem-se importantes nos processos de trabalho por permitir que os trabalhadores exerçam suas atividades de forma segura, mantendo sua integridade física e prevenindo-se de acidentes de trabalho ${ }^{19}$.

Com relação às condutas médicas, essas refletiram as necessidades dos trabalhadores e em sua grande maioria foram pertinentes aos encaminhamentos a algumas especialidades e em segundo lugar à solicitação de exames. Diante das queixas mais frequentes dos trabalhadores, que foram as ósseas, a maior parte das condutas médica voltaram-se ao encaminhamento desses trabalhadores ao atendimento ortopédico, atendendo às necessidades deles.

\section{CONCLUSÃO|}

O presente estudo possibilitou o conhecimento de características demográficas e clínicas importantes da classe trabalhadora atendida no CEREST Lagarto. Foi possível concluir que o referido centro atendeu, no período estabelecido pela pesquisa, uma população de trabalhadores em sua grande maioria do sexo masculino, naturais de Lagarto, com faixa etária entre 30 e 49 anos e tempo de serviço inferior a 10 anos. Bem como se concluiu que houve uma prevalência de lavradores entre as ocupações, com queixas ósseas em grande maioria, e que as condutas médicas se voltaram principalmente ao encaminhamento a especialidades, com destaque para a ortopedia.

Notou-se que o referido CEREST, embora seja de abrangência regional, necessita expandir seu atendimento para outros municípios, pois atualmente encontra-se limitado à cidade de Lagarto. Observa-se ainda que a normatização de registros ainda é muito deficiente e algumas variáveis importantes não foram analisadas devido à ausência de registros, o que constituiu um entrave para a contemplação de todos os dados que a pesquisa visava investigar.

Estudos com essa finalidade são de grande valia, pois ajudam a identificar as principais queixas clinicas, conhecer os principais tipos de atendimento médico local, além de prevenir potenciais agravos à saúde dos trabalhadores. É a partir de estudos de investigação que necessidades locais são identificadas e intervenções sociais são desenvolvidas a fim de melhorar o contexto em que a população local está inserida.

\section{REFERÊNCIAS |}

1. Brasil. Ministério da Saúde. Caderno de atenção básica: programa saúde da família (caderno 5: saúde do trabalhador). Brasília: Ministério da Saúde; 2002.

2. Brasil. Ministério da Saúde. Conselho Nacional de Secretários de Saúde. Vigilância em saúde: parte 1. Brasília: Ministério da Saúde; 2011.

3. Costa D, Lacaz FAC, Jackson Filho JM, Vilela RAG. Saúde do trabalhador no SUS: desafios para uma política pública. Rev Bras Saúde Ocup. 2013; 38(127):11-30.

4. Sousa-Uva A, Serranheira F. Trabalho e saúde/(doença): o desafio sistemático da prevenção dos riscos profissionais e o esquecimento reiterado da promoção da saúde. Rev Bras Med Trab. 2013; 11(1):43-9.

5. Brasil. Portaria no 1.679, de 19 de setembro de 2002. Dispõe sobre a estruturação da rede nacional de atenção integral à saúde do trabalhador no SUS e dá outras providências. Diário Oficial da União; 2002.

6. Brasil. Portaria n 2.728 , de 11 de novembro de 2009. Dispõe sobre a rede nacional de atenção integral à saúde do trabalhador (RENAST) e dá outras providências. Diário Oficial da União; 2009.

7. Jacques CC, Milanez B, Mattos RCOC. Indicadores para centros de referência em saúde do trabalhador: proposição 
de um sistema de acompanhamento de serviços de saúde. Ciênc Saúde Coletiva. 2012; 17(2):369-78.

8. Dias EC, Rigotto RM, Augusto LGS, Cancio J, Hoefel MGL. Saúde ambiental e saúde do trabalhador na atenção primária à saúde no SUS: oportunidades e desafios. Ciênc Saúde Coletiva. 2009; 14(6):2061-70.

9. Cazarin G, Augusto LGS, Melo RAM. Doenças hematológicas e situações de risco ambiental: a importância do registro para a vigilância epidemiológica. Rev Bras Epidemiol. 2007; 10(3):380-90.

10. Moreira JPL, Oliveira BLCA, Muzi CD, Cunha CLF, Brito AS, Luiz RR. A saúde dos trabalhadores da atividade rural no Brasil. Cad Saúde Pública. 2015; 31(8):1698-1708.

11. Oliveira ERSS, Costa JE. A pequena produção familiar no município de Lagarto/Sergipe. Rev Geog Am Central. 2011; 2(47):1-12.

12. Santana CM, Costa AR, Nunes RMP, Nunes NMF, Peron AP, Cavalcante AACM, et al. Exposição ocupacional de trabalhadores rurais a agrotóxicos. Cad Saúde Colet. 2016; 24(3):301-7.

13. Costa CKL, Lucena NMG, Tomaz AF, Másculo FS. Avaliação ergonômica do trabalhador rural: enfoque nos riscos laborais associados à carga física. GEPROS. 2011; (2):101-12.

14. Almeida MCS, Baptista PCP, Silva A. Cargas de trabalho e processo de desgaste em Agentes Comunitários de Saúde. Rev Esc Enferm USP. 2016; 50(1):95-103.

15. Lima AG, Silva AMM, Soares CEC, Souza RAX, Souza MCMR. Fotoexposição solar e fotoproteção de agentes de saúde em município de Minas Gerais. Rev Eletr Enf [Internet]. 2010 [acesso em 15 dez. 2016]; 12(3):478-82. Disponível em: URL: <http://dx.doi.org/10.5216/ree. v12i3.6156>.

16. Cezar-Vaz MR, Bonow CA, Piexak DR, Kowalczyk S, Vaz JC, Borges AM. Câncer de pele em trabalhadores rurais: conhecimento e intervenção de enfermagem. Rev Esc Enferm USP. 2015; 49(4):564-71.

17. Bedor CNG, Ramos LO, Pereira PJ, Rêgo MAV, Pavão AC, Augusto LGS. Vulnerabilidades e situações de riscos relacionados ao uso de agrotóxicos na fruticultura irrigada. Rev Bras Epidemiol. 2009; 12(1):39-49.

18. Mazon LM, Souza AZP. Estudo exploratório entre trabalhadores rurais expostos ocupacionalmente a agrotóxicos. Rev UNIANDRADE. 2015; 16(1):31-8.

19. Santos GNF, Neves JB. Equipamento de proteção individual: utilização pelos trabalhadores do setor de obras. Rev Enferm Integrada. 2015; 8(1):1325-34.

Correspondência para/Reprint request to:

Maria do Socorro Claudino Barreiro

Avenida Marechal Rondon, s/n,

Jardim Rosa Elre, São Cristóvão/SE, Brasil

CEP: 49100-000

E-mail:socorrocbarreiro@gmail.com

Submetido em: 14/09/2017

Aceito em: 22/11/2018 